\title{
Metacognitive awareness of covert somatosensory attention corresponds to contralateral alpha power
}

\author{
Stephen Whitmarsh ${ }^{\mathrm{a}, \mathrm{b}, *}$, Henk Barendregt ${ }^{\mathrm{b}}$, Jan-Mathijs Schoffelen ${ }^{\mathrm{a}}$, Ole Jensen ${ }^{\mathrm{a}}$ \\ a Donders Centre for Cognitive Neuroimaging, Box 9104, 6500 HE Nijmegen, The Netherlands \\ b Institute for Computing and Information Sciences, Radboud University Nijmegen, Box 9010, 6500 GL Nijmegen, The Netherlands
}

\section{A R T I C L E I N F O}

Article history:

Accepted 10 July 2013

Available online 17 July 2013

\section{Keywords:}

Attention

Metacognition

Neural oscillations

Metacognition

Alpha

Neural oscillations

Awareness

\begin{abstract}
A B S T R A C T
Studies on metacognition have shown that participants can report on their performance on a wide range of perceptual, memory and behavioral tasks. We know little, however, about the ability to report on one's attentional focus. The degree and direction of somatosensory attention can, however, be readily discerned through suppression of alpha band frequencies in EEG/MEG produced by the somatosensory cortex. Such top-down attentional modulations of cortical excitability have been shown to result in better discrimination performance and decreased response times. In this study we asked whether the degree of attentional focus is also accessible for subjective report, and whether such evaluations correspond to the amount of somatosensory alpha activity. In response to auditory cues participants maintained somatosensory attention to either their left or right hand for intervals varying randomly between 5 and 32 seconds, while their brain activity was recorded with MEG. Trials were terminated by a probe sound, to which they reported their level of attention on the cued hand right before probe-onset. Using a beamformer approach, we quantified the alpha activity in left and right somatosensory regions, one second before the probe. Alpha activity from contra- and ipsilateral somatosensory cortices for high versus low attention trials were compared. As predicted, the contralateral somatosensory alpha depression correlated with higher reported attentional focus. Finally, alpha activity two to three seconds before the probe-onset was correlated with attentional focus. We conclude that somatosensory attention is indeed accessible to metacognitive awareness.
\end{abstract}

(c) 2013 Elsevier Inc. All rights reserved.

\section{Introduction}

Metacognition refers to the general ability to reflect upon, and comment on mental states and cognitive processes. Traditionally, metacognition has been an important concept in understanding failure in memory performance such as false recognition and tip-of-the-tongue (for an overview see Metcalfe and Shimamura (1994) and Dunlosky and Bjork (2008)). More recently the concept of metacognition has been embraced by a broader range of cognitive neuroscience researchers. In cognitive tasks requiring a behavioral response, the ability to report confidence about one's performance has been used as paradigmatic example of metacognition (Fleming and Dolan, 2012). In the perceptual domain metacognitive reports are taken as reflecting conscious awareness of the percept, both in human (Kunimoto et al., 2001; Szczepanowski and Pessoa, 2007) and non-human research (Smith et al., 2012). However, in some cases metacognition might be simply understood as secondorder behavior, i.e. as behavior that is contingent on other (overt or

\footnotetext{
* Corresponding author at: Donders Centre for Cognitive Neuroimaging, Box 9104, 6500 HE Nijmegen, The Netherlands.

E-mail addresses: stephen.whitmarsh@gmail.com (S. Whitmarsh), henk@cs.ru.nl (H. Barendregt), j.schoffelen@donders.ru.nl (J.-M. Schoffelen), o.jensen@donders.ru.nl (O. Jensen).
}

nascent) behavior, rather than knowledge that is based on a metacognition (Fleming et al., 2012). This issue is particularly relevant in studies of attention, where so far metacognition has only been investigated within the context of behavioral performance. Such experimental paradigms permit metacognitive reports to be based on performance and stimulus processing, rather than on introspection of the cognitive (attentional) state. The primary objective of this study was therefore to show that attentional focus is metacognitive accessible, independently from the task performance or exogenous stimulus processing.

Neuroimaging techniques can disambiguate the metacognitive accessibility of attention by providing objective proxies of covert attention. Visuospatial and somatosensory attention can be gauged using magnetoencephalography (MEG) or electroencephalography (EEG) measurements of the $10 \mathrm{~Hz}$ rhythms found in the visual and somatosensory cortex (Hari and Salmelin, 1997; Pfurtscheller and Lopes da Silva, 1999). It is now a well-replicated finding that alpha activity decreases contralateral to the focus of attention, during visuospatial attention (Handel et al., 2011; Kelly et al., 2009; Rihs et al., 2007; Thut et al., 2006; van Gerven and Jensen, 2009; Worden et al., 2000) as well as during somatosensory attention (Haegens et al., 2011, 2012; Schubert et al., 2009; van Ede et al., 2010, 2011). Furthermore, visual and somatosensory alpha power have been shown to be modulated according to attentional demands (Gould et al., 2011; Haegens et al., 2011), affecting 
subsequent performance (Bengson et al., 2012; Haegens et al., 2011; Handel et al., 2011; Kelly et al., 2009; O'Connell et al., 2009; Thut et al., 2006). These findings show evidence for the attentional role of visual and somatosensory alpha through their role in augmenting and attenuating task relevant and irrelevant regions, respectively (Jensen and Mazaheri, 2010; Klimesch, 1999; Neuper and Pfurtscheller, 2001; Schack and Klimesch, 2002). In fact, combined EEG-fMRI studies have shown that posterior alpha power correlates negatively with visual BOLD activity (Scheeringa et al., 2011), while central alpha power correlates negatively with BOLD in somatosensory regions (Ritter et al., 2009). Taken together, alpha power has been shown to be sensitive both the degree as well as the location of covert visuospatial and somatosensory attention.

Previous work suggests that attention might be metacognitively accessible. In a recent EEG study, Macdonald et al. (2011) let participants report their level of attention during each trial in a visual discrimination task. Self-reported attention on task correlated negatively with prestimulus alpha power. In Braboszcz and Delorme (2011), subjects were instructed to count their breath and report by button-press whenever they noticed a distraction from the task. Posterior alpha and central beta power were shown to be reduced preceding these reports of mindwandering. These findings were interpreted in terms of impaired working-memory during mind-wandering. In Christoff et al. (2009), attention was sampled during a sustained attention task (SART, Robertson et al. (1997)). Moments of mind-wandering were shown not to be associated with any decreases of BOLD activity in task-related regions. Rather, they were reflected by a pattern of increased activity in both executive regions (dorsal ACC and the dorsolateral prefrontal cortex) and the default network (medial PFC, posterior cingulate and posterior temporo-parietal cortex), consistent with previous reports of default mode network activity during mind-wandering (Mason et al., 2007). Interestingly, this effect was found to be reduced when participants reported to have been aware of being distracted, suggesting that mind-wandering was most pronounced when it lacked metacognition. Taken together, these findings suggest that the attentional state during task performance might be metacognitively accessible. However, it remains an open question whether metacognition of attention can occur in the absence of a concurrent task.

The current study was designed to measure metacognition of attention independently from concurrent task performance and stimulus processing. Participants were instructed to try to maintain maximal attention to their left or right hand as indicated by auditory cues. At random periods after the cue, trials were terminated by a probe sound. A button-press was then used to self-report the degree in which attention was directed to the cued hand at the moment preceding the probe sound. These subjective self-reports were associated with alpha as an objective proxy of attention. For this purpose, MEG was used in combination with the beamformer method to estimate alpha power at the left and right somatosensory cortex. We hypothesized that trials with higher self-reported attention would be associated with lower alpha power in the contralateral somatosensory region. Confirmation of this hypothesis permitted us to conclude that the attentional focus is indeed metacognitively accessible.

\section{Methods}

\section{Participants}

Fifteen healthy participants ( 9 female, mean age 30.4 years, range: 19-63) enrolled after providing written informed consent and were paid in accordance with guidelines of the local ethics committee (CMO Committee on Research Involving Humans subjects, region ArnhemNijmegen, The Netherlands). One participant was excluded from the analysis due to excessive movement artifacts. The experiment was in compliance with national legislation as well as the code of ethical principles (Declaration of Helsinki).

\section{Experiment}

Participants were instructed to continuously attend to the cued hand while simultaneously trying to remain aware of their attentive state until a probe sound ( $2000 \mathrm{~Hz}$ tone) was presented (Fig. 1A). Cues consisted of two sequential tones of $400 \mathrm{~ms}$ each, $200 \mathrm{~ms}$ apart, with either ascending in pitch for the right hand $(2000 \mathrm{~Hz}$ followed by $2500 \mathrm{~Hz}$ ) or descending for the left hand $(2000 \mathrm{~Hz}$ followed by $1500 \mathrm{~Hz}$ ). Cue side was determined pseudo-randomly. Cue-probe intervals followed an exponential distribution with a mean of 3 seconds and a cut-off time of 27 seconds, providing a flat hazard rate. In other words, the chance of the probe occurring after trial onset was held constant. A minimal cue-probe interval of 5 seconds was added, resulting in an average cue-probe interval of 8 seconds and maximal of 32 seconds. After the probe sound, participants evaluated their level of attention on the cued hand using one out of four options: (1) not at all, (2) little, (3) much, (4) fully/maximally attentive. The experiment started with a training session, followed by three continuous blocks of 125 trials separated by self-paced breaks. The response hand at the first session was determined randomly, and then switched for each block. To minimize head movements and provide comfort, participants were measured in supine position. To minimize eye movements and blinks and increase the chance of fluctuations in attentional focus, participants were instructed to remain with their eyes closed throughout the experiment.

\section{Data preprocessing}

Continuous MEG data were recorded using a 275-sensor axial gradiometer system (CTF MEG TM Systems Inc., Port Coquitlam, BC, Canada) placed in a magnetically shielded room. The ongoing MEG signals were low-pass filtered at $300 \mathrm{~Hz}$, digitized at $1200 \mathrm{~Hz}$, and stored for off-line analysis. The subjects' head position was continuously recorded relative to the gradiometer array using coils positioned at the subject's nasion and at the left and right ear canals. High-resolution anatomical images ( $1 \mathrm{~mm}$ isotropic voxel size) were acquired using a 1.5 -T Siemens Magnetom Sonata system (Erlangen, Germany). The same earplugs, using vitamin $\mathrm{E}$ instead of the coils, were used for co-registration with the MEG data. MEG data was analyzed using the Matlab-based Fieldtrip toolbox, developed at the Donders Institute for Brain, Cognition and Behavior (Oostenveld et al., 2011). Trials containing movement, muscle, and superconducting quantum interference device (SQUID) jumps were discarded by visual inspection. Independent component analysis (ICA) was used to remove eye and heart artifacts.

\section{Source reconstruction of alpha power}

Source reconstruction was performed using a frequency-domain beamformer approach (Dynamic Imaging of Coherent Sources) which uses adaptive spatial filters to localize power in the entire brain (Gross et al., 2001; Liljestrom et al., 2005). The brain volume of each individual subject was discretized to a grid with a $0.8-\mathrm{cm}$ resolution. For every grid point a spatial filter was constructed from the cross-spectral density matrix and the lead field. The lead fields were calculated from a subject specific realistic single-shell model of the brain (Nolte, 2003), based on the individual anatomical MRIs. We calculated the cross-spectral density matrix based upon the full interval between cue offset and probe onset to obtain the most accurate estimation of the alpha sources. Individual alpha frequencies were used for each subject (for all grid and time points), determined by the maximum log power between 7 and $15 \mathrm{~Hz}$ on all trials and sensors.

For each grid point and six one-second time segment preceding probe onset, alpha activity was then estimated. A sufficient number of trials $(\sim 100)$ had trial lengths of at least 6 seconds preceding probe onset to enable source statistics at those intervals. A (Slepian) multitaper approach was used to accomplish accurate frequency smoothing ( $\pm 2 \mathrm{~Hz}$ ) around the subject-specific alpha peaks. To enable 
A

paradigm

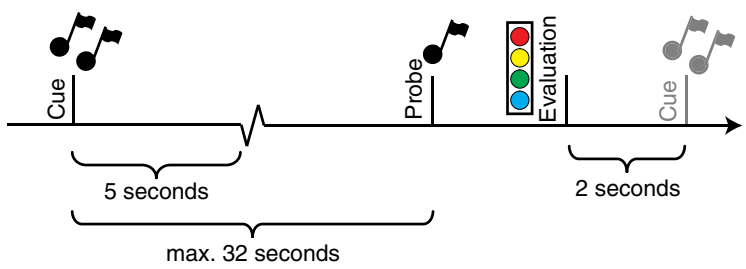

B

\section{example design matrix}

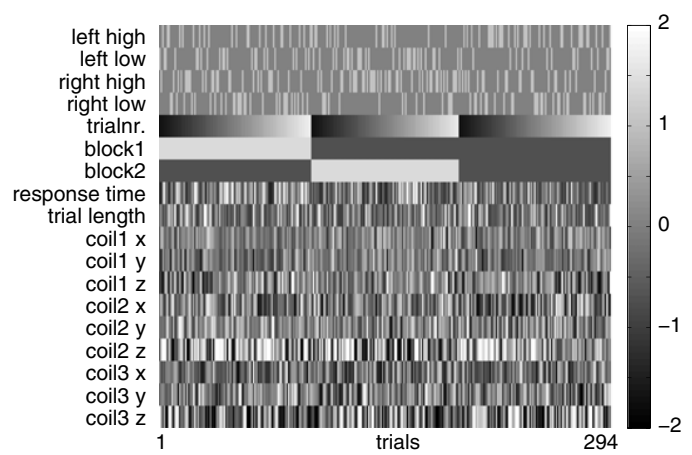

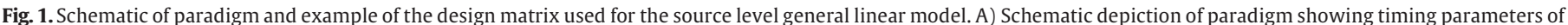
trial. B) Example design matrix showing regressors for conditions (0's and 1's) and confound regressors (normalized).

valid voxel-by-voxel comparisons in the face of the beamformer depth bias, alpha estimates were standardized over trials.

\section{Source level GLM}

A voxel-by-voxel first-level GLM approach was then used for every subject and time segment. Fig. 1B shows an example design matrix, including cue side (left/right) and self-report (high/low), dichotomized according to a median split per subject and time-point. In addition, trial number and the mean $\mathrm{X}, \mathrm{Y}$ and $\mathrm{Z}$ position of the three fiducial coils were entered as separate regressors. The locations of the fiducial coils indicate the position of anatomical landmarks of the subject's head (nasion and pre-auricular points) in the MEG helmet. Regressors based on fiducial coil positions can therefore be used to subtract variance caused by differences in head position over trials (Stolk et al., 2013). To further reduce variance that could be explained by response preparation, regressors for evaluation response times and cue-probe duration were added to the GLM, together with separate regressors for the response hand, which was switched between each block and randomized over subjects. The cue side and self-report predictors consisted of 0 's and 1 's, thereby yielding mean standardized alpha power after multiplication with the standardized data. By standardizing the remaining covariates (response time, trial length, fiducial position, etc.) multiplication with the standardized data resulted in correlation values $(r)$. Prior to averaging and group statistics, the resulting $\beta$-values and correlations values were spatially normalized using SPM2 to the International Consortium for Brain Mapping template (Montreal Neurological Institute, MNI, Montreal, QC, Canada).

\section{Functional localization of primary somatosensory regions}

After the reconstruction of alpha power for each voxel and timepoint, somatosensory regions of interest (ROIs) were determined based on alpha power during the last second preceding probe onset. A voxel-by-voxel comparison was made between left and right attention trials. A cluster-based permutation test (Maris and Oostenveld, 2007) was then used to identify significant spatial clusters. This resulted in a distinct somatosensory alpha-ROI for each hemisphere. Each ROI therefore depended on cue condition (left versus right), but remained independent of the self-reported evaluation of attention.

\section{Region of interest analysis}

Alpha power values within the left and right ROI voxels were averaged according to cue condition (ipsi versus contra), evaluation (high versus low) and time-point (six one-second intervals preceding probe onset). The effects of cue condition and evaluation on mean alpha power were tested over time using repeated measures ANOVA. Differences in these effects over time were tested using post-hoc $t$-tests per time-point.

\section{Results}

Participants were instructed to try to maintain maximal attention to either their left or right hand as indicated by auditory cues. At random periods after the cue, trials were terminated by another (probe) sound. After each trial participants reported by button-press ( 1 to 4 ) the degree of attention that was allocated to the cued hand at the moment right before the probe sound.

\section{Behavior}

Attentional focus fluctuated over time, as reflected by the use of the full range of responses (Fig. 2A). The number of responses per evaluation, differed significantly $(F(3)=9.896, p<0.001)$, showing a linear relationship $(F(1)=24.778, p<0.001)$, with evaluations being generally high. This shows that participants were confident about their performance. Evaluation times also differed for the different levels (Fig. 2B; $F(3)=28.739, p<0.001$ ). Evaluations that were rated high were also made quicker, showing again a linear trend $(F(1)=47.133$, $p<0.001$ ). Furthermore, evaluation times correlated negatively with cue-probe duration (mean $\mathrm{r}=-0.130, \mathrm{t}(13)=-5.5048, p=<0.001$ ), showing that longer cue-probe durations did not result in a loss of vigilance or ability to do the task.

\section{Functional localization of primary somatosensory regions}

Somatosensory alpha regions of interest (ROIs) were determined on the basis of the distribution of alpha power in the brain volume estimate using the beamformer approach applied to the MEG data during the last second before probe onset. A cluster-based permutation test (Maris and Oostenveld, 2007) was used to identify the significant clusters responsive to cue direction. The analysis resulted in two significant clusters, one in each hemisphere in primary sensorimotor areas (see Fig. 3). These ROIs were used for further analysis of the alpha power preceding metacognitive evaluations. For this purpose estimates of alpha power in the left and right hemispheric ROI's were separated into an ipsi-lateral ROI and a contra-lateral ROI on the basis of hemisphere and cue direction. In other words, ipsi-lateral alpha power consisted of the left ROI during left-attention trials, and the right ROI during right-attention trials. Similarly, contra-lateral alpha power consisted of the left ROI during right-attention trials and the right ROI during left-attention trials. The ipsi-contra distinction was therefore independent of the distinction in left and right hemispheric ROI. 
Regions of interest analysis

Alpha power was first averaged over grid points in the left and right ROI and over the 6 seconds interval preceding probe onset. As predicted, differences in self-reported evaluation corresponded to differences in preceding alpha power, showing lower alpha power for high attention versus low attention $(F(1,13)=7.163, p=0.019$; compare top panel with bottom panel in Figs. $4 A$ and $B$ ). We then went on to analyze alpha power preceding probe onset in a post-hoc manner, to investigate the temporal profile of alpha activity preceding probe onset (Figs. $4 \mathrm{C}$ and $\mathrm{D}$, respectively). Only the contralateral ROI showed significant differences between high and low evaluations, for the last 3 seconds preceding probe onset (two-tailed: $[-3:-2] t(13)=-2.357$, $p=0.035 ;[-2:-1] t(13)=0.017 ;[-1: 0] t(13)=-2.648, p=$ $0.020)$.

\section{Confound regressors analysis}

Control regressors were used to control for variance due to potential differences in response times, cue-probe duration, elapsed time (trial number) and movement (3D position of fiducial coils). These regressors were standardized and entered as covariates in the design matrix. Resultant correlation values $(r)$ were tested against zero using clusterbased permutation tests (Maris and Oostenveld, 2007). Cue-probe duration was shown to be significantly positively correlated with superior-parietal alpha power, and this correlation was shown to be maintained over time (Fig. 5A). This means that alpha activity in these regions gradually increased as the trial became longer. Response time correlated with some alpha activity at somatosensory and visual regions, although these effects did not remain significance for any extended period of time (Fig. 5B). In conclusion, the level of subjective attention was mainly reflected by contralateral alpha in somatosensory regions and was not confounded by correlations with response times or cue-probe interval.

\section{Discussion}

The main purpose of this study was to investigate whether the degree of somatosensory attentional focus is metacognitively accessible, as shown by a correspondence between contralateral alpha and selfreported attentional focus. As a prerequisite for our study, alpha activity produced in primary somatosensory regions was first shown to be hemispherically lateralized in response to cued attention to the left or right hand. Furthermore, by demonstrating significant lateralization in the somatosensory cortex during the second preceding probe onset, it was shown that this lateralization can be sustained for extended periods of time (from 8 to 32 seconds). The main finding was that participants' subjective rating of their attentional focus was reflected in somatosensory alpha power contralateral to the attended hand. This somatotopic specificity supports the notion that participants were not reporting on
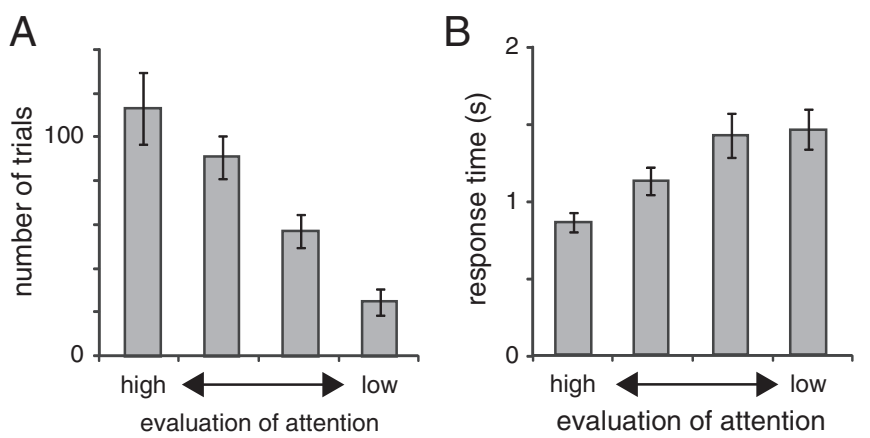

Fig. 2. Behavioral differences between evaluation responses. A) Distribution of trials according to evaluation of attention shows that attention was generally rated high. B) Evaluation times were reduced when attention was evaluated higher.
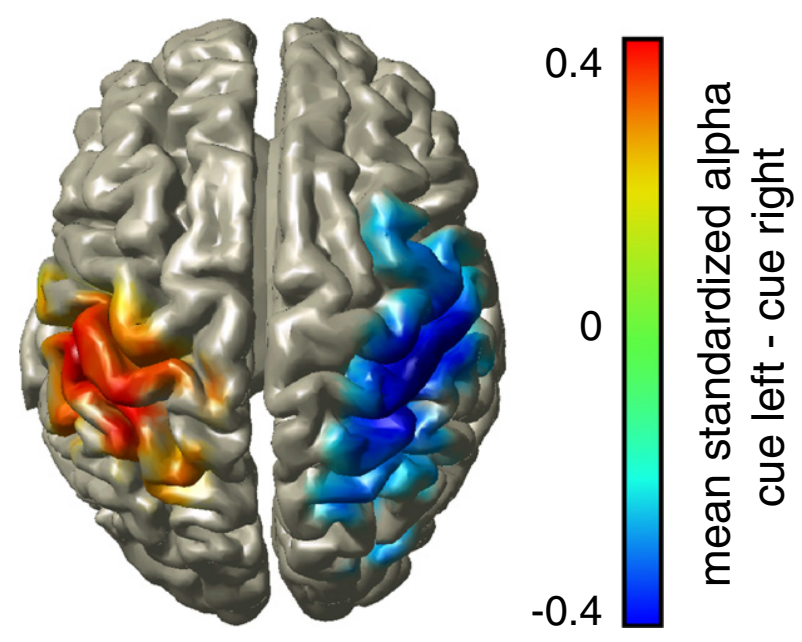

Fig. 3. Somatosensory alpha power lateralized in response to cue direction. Source reconstructed alpha activity during one second preceding probe-onset shows clear lateralization at primary somatosensory regions. Significant voxels were thresholded based on cluster-based permutation test (Maris and Oostenveld, 2007).

a general attentional state, but were able to report specifically on their attentional sensory specific focus. This study therefore provides strong support for the metacognitive accessibility of attentional focus. Moreover, this is the first study showing metacognition in the absence of either exogenous stimulation or evaluations of task performance based on overt behavior. Furthermore, response times were shown not to contribute to somatosensory alpha power, supporting the behavioral independence of our findings.

Our findings are consistent with previous electrophysiological studies on metacognition. A previous investigation by Braboszcz and Delorme (2011) showed reduced posterior alpha during mind-wandering, which the authors interpreted in terms of impaired working-memory processes. However, the role of alpha oscillations during working memory has also been understood to reflect active inhibition of task-unrelated activity, suppressing visual processes during memory retention (Jensen and Mazaheri, 2010; Klimesch, 1999; Neuper and Pfurtscheller, 2001; Schack and Klimesch, 2002). The results of Braboszcz and Delorme (2011) could therefore have reflected mal-adaptive attention to visual processes during mind-wandering. This would put their findings in line with ours, providing converging evidence for the metacognitive accessibility of the internal attentional state. A recent sophisticated EEG study by Macdonald et al. (2011) found a negative correlation between prestimulus parieto-occipital alpha power and self-reported attention during a visual detection task. Interestingly, parieto-occipital alpha and self-reported attention correlated over periods of several minutes. Such slow fluctuations of attention are in accordance with O'Connell et al. (2009), where lapses in visual attention were preceded by increased parieto-occipital alpha for at least 20 seconds before an error occurred. Importantly, neither O'Connell et al. (2009) nor Macdonald et al. (2011) used cued spatial attention, but rather correlated self-reports with the measurement of general visual attention. Our data extend these findings by showing that self-reported attentional focus corresponds most strongly with contra-lateral (to cued side) somatosensory alpha. This demonstrates that the correlation between self-report and alpha activity can be spatially specific. In other words, while the findings by O'Connell et al. (2009) and Macdonald et al. (2011) suggest metacognitive access to the visual attentional state, our findings provide strong evidence for metacognitive access to spatial focus as well.

Previous work has shown that, in visuospatial (Fu et al., 2001; Kelly et al., 2006; Worden et al., 2000) and somatosensory attention tasks (Haegens et al., 2012), the inclusion of distracting stimuli at the uncued side can result in an increase of ipsilateral alpha power, reflecting 
A

CONTRA-LATERAL

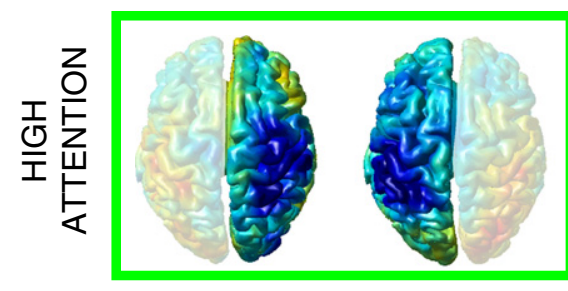

$\Rightarrow$ CUE $\Rightarrow$

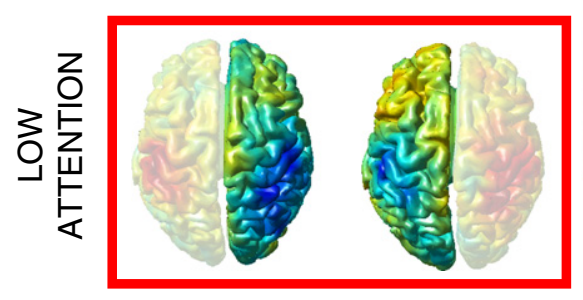

C

CONTRA-LATERAL

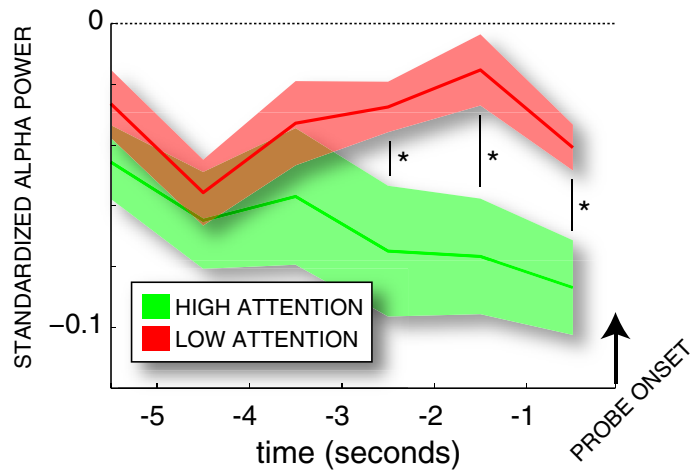

B

IPSI-LATERAL

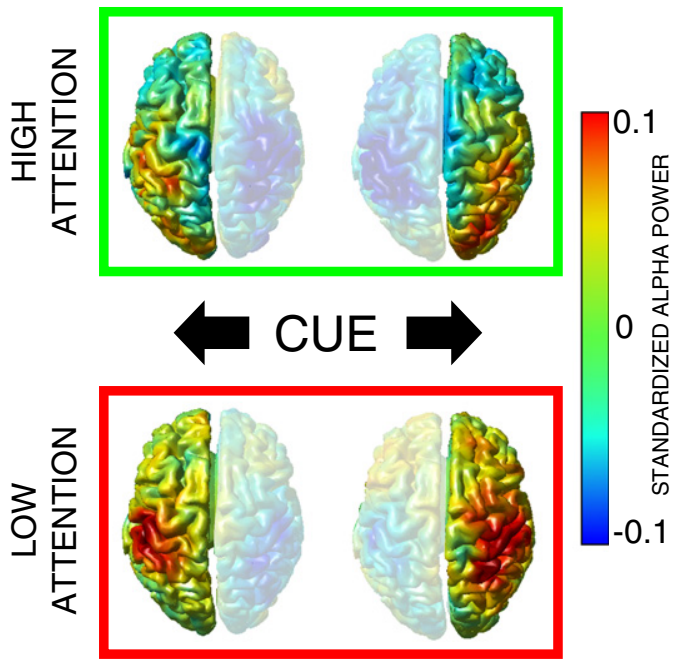

$\mathrm{D}$

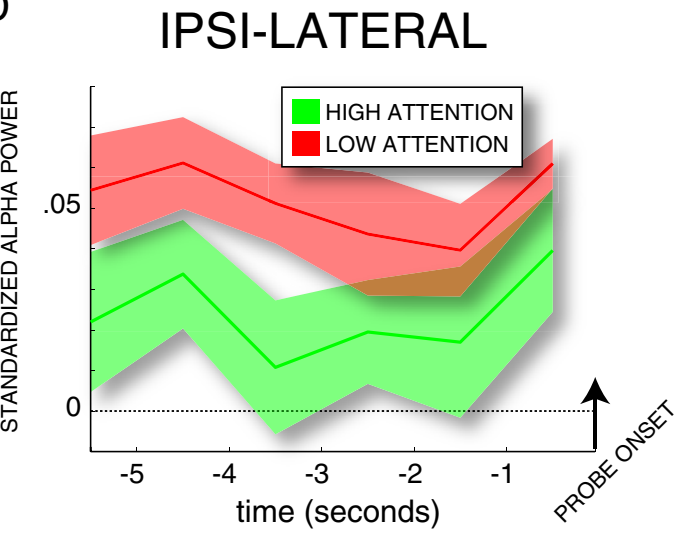

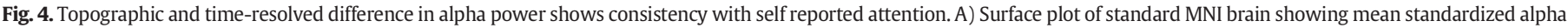

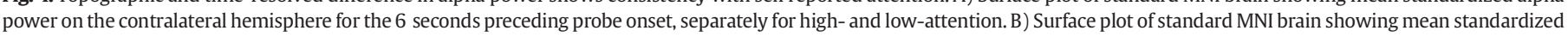

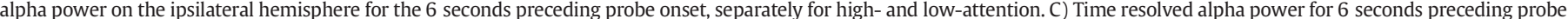

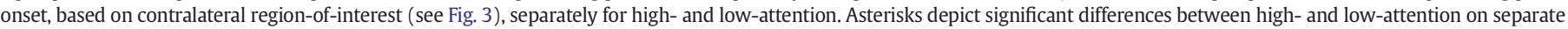

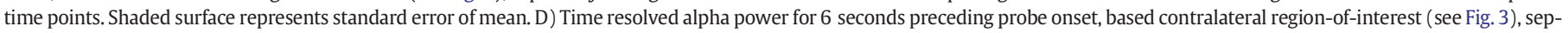
arately for high- and low-attention. Curve width represents standard error of mean.

active suppression of the distracting sensations. In the current experiment, participants were only required to attend continuously to the cued hand while no distractors were presented. Consistent with the idea that sensations from the un-cued hand posed little challenge and did not need to be actively inhibited, alpha power tended to be lower rather than higher at the ipsilateral hemisphere when attention was reported to be higher, although they did not do so significantly. This suggests that metacognitive reports were not based on the unattended hand. Future experiments, however, could investigate the potential to not only report on the degree of attention, but also on the degree of suppression of distraction.

Interestingly, in our analysis of confound regressors, cue-probe duration was found to correlate positively with superior parietal alpha power at regions. The superior parietal lobule (SPL), in particular the inferior parietal sulcus (IPS), has been implicated in body-centered coding (Galati et al., 2010) and movement preparation (Cohen and Andersen, 2002). Furthermore, the SPL/IPS is considered part of the Dorsal Attention Network (DAN; Corbetta and Shulman (2002, 2011)), involved in goal-directed orientation of attention. Not much is known about the role of SPL alpha oscillations, however. The question remains if superior-parietal alpha has similar inhibitory effects as in sensory regions (Jensen and Mazaheri, 2010; Klimesch, 1999; Neuper and
Pfurtscheller, 2001; Schack and Klimesch, 2002). A recent study on parietal alpha of local field potentials in the macaque monkey (Premereur et al., 2012) does suggest that parietal alpha actively inhibits the onset of target-oriented saccades, consistent with the involvement of parietal alpha in encoding gaze-centered reference-frames (Buchholz et al., 2011; Van Der Werf et al., 2012). As a matter of speculation, our results could therefore suggest increased suppression of reorienting activity with increasing trial duration.

Decades ago, Nelson and Narens (1994) argued eloquently for metacognition as a topic of interest in its own right as well as a bridge between many areas of cognitive and psychological investigation, e.g., between decision making and memory, learning and motivation. Recently, action and perceptual processes have been added to this list (see Fleming et al. (2012) for an overview) and with our and other recent studies (Braboszcz and Delorme, 2011; Christoff et al., 2009; Macdonald et al., 2011), attention and mind-wandering as well. This speaks to the general role of metacognition as cognitive processes that monitor and control cognition (cf. e.g., Fernandez-Duque et al., 2000). According to metacognitive principles, cognition can be split into two interrelated levels: the metalevel and the object level (Nelson and Narens, 1990). While the metalevel is continuously updated by bottom-up information, it asserts controls over the object level by 
A
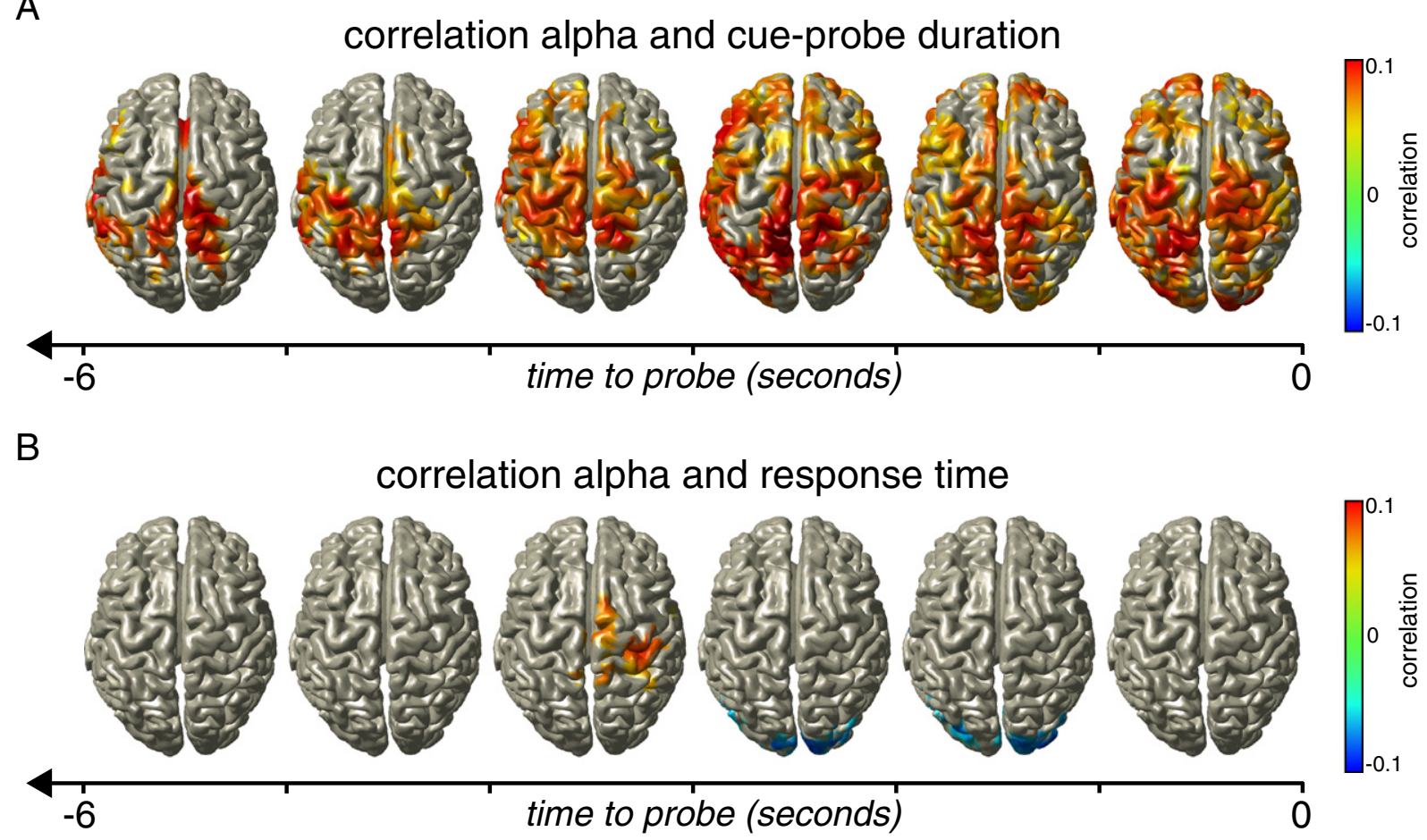

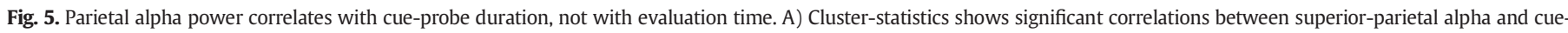
probe duration, consistent over time before probe-onset. B) No consistent correlations between alpha power and evaluation times were found.

providing top-down input and initiating or terminating its actions (Nelson and Narens, 1990). In this light, our measure of somatosensory attention reflects the subservient object level rather than the metalevel. Our paradigm did not enable a valid comparison between trials in which metacognition was present and trials where it was absent. However, a recent study on interoception of event-times (Guggisberg et al., 2011) has done so. Interestingly, the patterns of neural activity that was related to introspection depended on the target of introspection, i.e., whether it concerned auditory perception, intentional or motor events. Each was related to a specific introspection-related network. Along similar lines, future studies can be expected to delineate frontal brain networks that are specifically involved in metacognitive monitoring of attention. Such research could take into account the rich literature on executive control in metacognition (see e.g., Botvinick et al. (2001) and Fernandez-Duque et al. (2000)) as well as recent models of attention that organize brain networks in terms of their relationship to internal goals and external cues (e.g., Corbetta and Shulman (2002) and Miller and D'Esposito (2005)). Our data suggest that part of the introspective process might involve the maintenance of attention through the suppression of reorienting responses arising from the Dorsal Attention Network (Corbetta and Shulman, 2002, 2011).

\section{Conflict of interest}

The authors report no financial conflict of interest with regards to the content of this paper.

\section{References}

Bengson, J.J., Mangun, G.R., Mazaheri, A., 2012. The neural markers of an imminent failure of response inhibition. Neuroimage 59 (2), 1534-1539.

Botvinick, M.M., Braver, T.S., Barch, D.M., Carter, C.S., Cohen, J.D., 2001. Conflict monitoring and cognitive control. Psychol. Rev. 108 (3), 624-652.

Braboszcz, C., Delorme, A., 2011. Lost in thoughts: neural markers of low alertness during mind wandering. Neuroimage 54 (4), 3040-3047.
Buchholz, V.N., Jensen, O., Medendorp, W.P., 2011. Multiple reference frames in cortical oscillatory activity during tactile remapping for saccades. J. Neurosci. 31 (46), 16864-16871.

Christoff, K., Gordon, A.M., Smallwood, J., Smith, R., Schooler, J.W., 2009. Experience sampling during fMRI reveals default network and executive system contributions to mind wandering. Proc. Natl. Acad. Sci. U. S. A. 106 (21), 8719-8724.

Cohen, Y.E., Andersen, R.A., 2002. A common reference frame for movement plans in the posterior parietal cortex. Nat. Rev. Neurosci. 3 (7), 553-562.

Corbetta, M., Shulman, G.L., 2002. Control of goal-directed and stimulus-driven attention in the brain. Nat. Rev. Neurosci. 3 (3), 201-215.

Corbetta, M., Shulman, G.L., 2011. Spatial neglect and attention networks. Annu. Rev. Neurosci. 34, 569-599.

Dunlosky, J., Bjork, R.A., 2008. Handbook of Metamemory and Memory. Psychology Press, New York.

Fernandez-Duque, D., Baird, J.A., Posner, M.I., 2000. Executive attention and metacognitive regulation. Conscious. Cogn. 9 (2), 288-307.

Fleming, S.M., Dolan, R.J., 2012. The neural basis of metacognitive ability. Philos. Trans. R. Soc. Lond. B Biol. Sci. 367 (1594), 1338-1349.

Fleming, S.M., Dolan, R.J., Frith, C.D., 2012. Metacognition: computation, biology and function Introduction. Phil. Trans. R. Soc. B Biol. Sci. 367 (1594), 1280-1286.

Fu, K.M., Foxe, J.J., Murray, M.M., Higgins, B.A., Javitt, D.C., Schroeder, C.E., 2001. Attentiondependent suppression of distracter visual input can be cross-modally cued as indexed by anticipatory parieto-occipital alpha-band oscillations. Brain Res. Cogn. Brain Res. 12 (1), 145-152.

Galati, G., Pelle, G., Berthoz, A., Committeri, G., 2010. Multiple reference frames used by the human brain for spatial perception and memory. Exp. Brain Res. 206 (2), $109-120$.

Gould, I.C., Rushworth, M.F., Nobre, A.C., 2011. Indexing the graded allocation of visuospatial attention using anticipatory alpha oscillations. J. Neurophysiol. 105 (3), 1318-1326.

Gross, J., Kujala, J., Hamalainen, M., Timmermann, L., Schnitzler, A., Salmelin, R., 2001. Dynamic imaging of coherent sources: studying neural interactions in the human brain. Proc. Natl. Acad. Sci. U. S. A. 98 (2), 694-699.

Guggisberg, A.G., Dalal, S.S., Schnider, A., Nagarajan, S.S., 2011. The neural basis of eventtime introspection. Conscious. Cogn. 20 (4), 1899-1915.

Haegens, S., Handel, B.F., Jensen, O., 2011. Top-down controlled alpha band activity in somatosensory areas determines behavioral performance in a discrimination task J. Neurosci. 31 (14), 5197-5204.

Haegens, S., Luther, L., Jensen, O., 2012. Somatosensory anticipatory alpha activity increases to suppress distracting input. J. Cogn. Neurosci. 24 (3), 677-685.

Handel, B.F., Haarmeier, T., Jensen, O., 2011. Alpha oscillations correlate with the successful inhibition of unattended stimuli. J. Cogn. Neurosci. 23 (9), 2494-2502.

Hari, R., Salmelin, R., 1997. Human cortical oscillations: a neuromagnetic view through the skull. Trends Neurosci. 20 (1), 44-49. 
Jensen, O., Mazaheri, A., 2010. Shaping functional architecture by oscillatory alpha activity: gating by inhibition. Front. Hum. Neurosci. 4, 186.

Kelly, S.P., Lalor, E.C., Reilly, R.B., Foxe, J.J., 2006. Increases in alpha oscillatory power reflect an active retinotopic mechanism for distracter suppression during sustained visuospatial attention. J. Neurophysiol. 95 (6), 3844-3851.

Kelly, S.P., Gomez-Ramirez, M., Foxe, J.J., 2009. The strength of anticipatory spatial biasing predicts target discrimination at attended locations: a high-density EEG study. Eur. J. Neurosci. 30 (11), 2224-2234.

Klimesch, W., 1999. EEG alpha and theta oscillations reflect cognitive and memory performance: a review and analysis. Brain Res. Brain Res. Rev. 29 (2-3), 169-195.

Kunimoto, C., Miller, J., Pashler, H., 2001. Confidence and accuracy of near-threshold discrimination responses. Conscious. Cogn. 10 (3), 294-340.

Liljestrom, M., Kujala, J., Jensen, O., Salmelin, R., 2005. Neuromagnetic localization of rhythmic activity in the human brain: a comparison of three methods. Neuroimage 25 (3), 734-745.

Macdonald, J.S., Mathan, S., Yeung, N., 2011. Trial-by-trial variations in subjective attentional state are reflected in ongoing prestimulus EEG alpha oscillations. Front. Psychol. 2, 82

Maris, E., Oostenveld, R., 2007. Nonparametric statistical testing of EEG- and MEG-data. J. Neurosci. Methods 164 (1), 177-190.

Mason, M.F., Norton, M.I., Van Horn, J.D., Wegner, D.M., Grafton, S.T., Macrae, C.N., 2007. Wandering minds: the default network and stimulus-independent thought. Science 315 (5810), 393-395.

Metcalfe, J., Shimamura, A.P., 1994. Metacognition: Knowing about Knowing. MIT Press, Cambridge, Mass.

Miller, B.T., D'Esposito, M., 2005. Searching for "the top" in top-down control. Neuron 48 (4), 535-538.

Nelson, T.O., Narens, L., 1990. Metamemory: A Theoretical Framework and New Findings. In: Bower, G. (Ed.), The psychology of learning and motivation. Academic Press, New York

Nelson, T.O., Narens, L., 1994. Why investigate metacognition? In: Metcalfe, J., Shimamura, A.P. (Eds.), Metacognition: Knowing about Knowing. MIT Press/Bradford Books, Cambridge.

Neuper, C., Pfurtscheller, G., 2001. Event-related dynamics of cortical rhythms: frequencyspecific features and functional correlates. Int. J. Psychophysiol. 43 (1), 41-58.

Nolte, G., 2003. The magnetic lead field theorem in the quasi-static approximation and its use for magnetoencephalography forward calculation in realistic volume conductors. Phys. Med. Biol. 48 (22), 3637-3652.

O'Connell, R.G., Dockree, P.M., Robertson, I.H., Bellgrove, M.A., Foxe, J.J., Kelly, S.P., 2009 Uncovering the neural signature of lapsing attention: electrophysiological signals predict errors up to $20 \mathrm{~s}$ before they occur. J. Neurosci. 29 (26), 8604-8611.

Oostenveld, R., Fries, P., Maris, E., Schoffelen, J.M., 2011. FieldTrip: open source software for advanced analysis of MEG, EEG, and invasive electrophysiological data. Comput. Intell. Neurosci. 2011, 156869.

Pfurtscheller, G., Lopes da Silva, F.H., 1999. Event-related EEG/MEG synchronization and desynchronization: basic principles. Clin. Neurophysiol. 110 (11), 1842-1857.
Premereur, E., Vanduffel, W., Janssen, P., 2012. Local field potential activity associated with temporal expectations in the macaque lateral intraparietal area. J. Cogn. Neurosci. 24 (6), 1314-1330.

Rihs, T.A., Michel, C.M., Thut, G., 2007. Mechanisms of selective inhibition in visual spatial attention are indexed by alpha-band EEG synchronization. Eur. J. Neurosci. 25 (2), 603-610.

Ritter, P., Moosmann, M., Villringer, A., 2009. Rolandic alpha and beta EEG rhythms' strengths are inversely related to fMRI-BOLD signal in primary somatosensory and motor cortex. Hum. Brain Mapp. 30 (4), 1168-1187.

Robertson, I.H., Manly, T., Andrade, J., Baddeley, B.T., Yiend, J., 1997. ‘Oops!’: performance correlates of everyday attentional failures in traumatic brain injured and normal subjects. Neuropsychologia 35 (6), 747-758.

Schack, B., Klimesch, W., 2002. Frequency characteristics of evoked and oscillatory electroencephalic activity in a human memory scanning task. Neurosci. Lett. 331 (2), 107-110.

Scheeringa, R., Fries, P., Petersson, K.M., Oostenveld, R., Grothe, I., Norris, D.G., et al., 2011. Neuronal dynamics underlying high- and low-frequency EEG oscillations contribute independently to the human BOLD signal. Neuron 69 (3), 572-583.

Schubert, R., Haufe, S., Blankenburg, F., Villringer, A., Curio, G., 2009. Now you'll feel it, now you won't: EEG rhythms predict the effectiveness of perceptual masking. J. Cogn. Neurosci. 21 (12), 2407-2419.

Smith, J.D., Couchman, J.J., Beran, M.J., 2012. The highs and lows of theoretical interpretation in animal-metacognition research. Philos. Trans. R. Soc. Lond. B Biol. Sci. 367 (1594), 1297-1309.

Stolk, A., Todorovic, A., Schoffelen, J.M., Oostenveld, R., 2013. Online and offline tools for head movement compensation in MEG. Neuroimage 68, 39-48.

Szczepanowski, R., Pessoa, L., 2007. Fear perception: can objective and subjective awareness measures be dissociated? J. Vis. 7 (4), 10.

Thut, G., Nietzel, A., Brandt, S.A., Pascual-Leone, A., 2006. Alpha-band electroencephalographic activity over occipital cortex indexes visuospatial attention bias and predicts visual target detection. J. Neurosci. 26 (37), 9494-9502.

Van Der Werf, J., Buchholz, V.N., Jensen, O., Medendorp, W.P., 2012. Reorganization of oscillatory activity in human parietal cortex during spatial updating. Cereb. Cortex 23 (3), 508-519.

van Ede, F., Jensen, O., Maris, E., 2010. Tactile expectation modulates pre-stimulus betaband oscillations in human sensorimotor cortex. Neuroimage 51 (2), 867-876.

van Ede, F., de Lange, F., Jensen, O., Maris, E., 2011. Orienting attention to an upcoming tactile event involves a spatially and temporally specific modulation of sensorimotor alpha- and beta-band oscillations. J. Neurosci. 31 (6), 2016-2024.

van Gerven, M., Jensen, O., 2009. Attention modulations of posterior alpha as a control signal for two-dimensional brain-computer interfaces. J. Neurosci. Methods 179 (1), 78-84.

Worden, M.S., Foxe, J.J., Wang, N., Simpson, G.V., 2000. Anticipatory biasing of visuospatial attention indexed by retinotopically specific alpha-band electroencephalography increases over occipital cortex. J. Neurosci. 20 (6), RC63. 